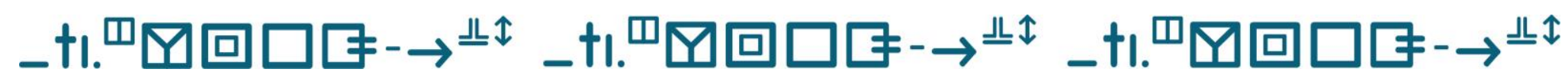

\section{Bullying, apoio social e sentido de vida: relato de discentes surdas}

\section{Bullying, social support and sense of life: reports of deaf discents}

\footnotetext{
(D) Valéria Maria Azevedo Guimarães

Universidade Federal de Sergipe, Aracaju, Sergipe, Brasil guimaraes.psicologa@gmail.com

iD 9 Karine David Andrade Santos

Universidade Federal de Sergipe, Aracaju, Sergipe, Brasil psimulti@gmail.com

iD 9

Nadja Pereira Dantas

Universidade Federal de Sergipe, Aracaju, Sergipe, Brasil nadjadantas2005@yahoo.com.br

Resumo: Este artigo tem por objetivo descrever as experiências de bullying vivenciadas por uma população surda no ambiente escolar e o papel do apoio social e do sentido de vida na trajetória familiar, laboral e acadêmica dessa população. Participaram desta pesquisa discentes surdas de uma instituição de ensino superior, que responderam a um roteiro de entrevista semiestruturada, cujas informações coletadas foram analisadas pelo software IRAMUTEQ. Os resultados foram apresentados por classes, a saber: apoio social e inclusão acadêmica, sentido de vida, relação familiar e bullying na escola. Verificou-se, nessas classes, que os surdos encontravam apoio social e emocional com os seus pares no ambiente universitário, construíam 
metas e projetos de vida para o futuro como uma via de realização existencial, vivenciavam obstáculos de comunicação no espaço familiar e, no período escolar, foram alvos de práticas de bullying devido à perda auditiva.

Palavras-chave: Surdez. Bullying. Apoio Social. Sentido de Vida.

Abstract: This article aims to describe the experiences of bullying experienced by a deaf population in the school environment and the role of social support and the meaning of life in the family, work, and academic trajectory of this population. Deaf students from a higher education institution participated in this research, responding to a semi-structured interview script, whose collected information was analyzed using the IRAMUTEQ software. The results were presented by class, namely: social support and academic inclusion, meaning of life, family relationship and bullying at school. It was found, in these classes, that the deaf found social and emotional support with their peers in the university environment, built goals and life projects for the future as a way of existential realization, experienced communication obstacles in the family space and, in the period at school, were targets of bullying practices due to hearing loss.

Keywords: Deafness. Bullying. Social Support. Meaning of Life.

Submetido em 08 de janeiro de 2021. Aceito em 07 de abril de 2021.

Publicado em 19 de novembro de 2021. 


\section{_tı.}

Bullying, apoio social e senso de vida: relato de discentes surdas

Valéria Maria Azevedo Guimarães • Karine David Andrade Santos et al.

\section{Introdução}

A ideia de que os indivíduos agem de acordo com suas próprias crenças está diretamente relacionada ao conceito de autoeficácia, que é definida como o julgamento que ele faz sobre suas habilidades e capacidades para atuar em um domínio específico, constituindo-se em alicerce para a motivação, o bem-estar e as futuras realizações pessoais (BANDURA, 1986, 1993, 1997). A forma de agir de acordo com as suas autocrenças Ihes permite assumir um determinado grau de controle sobre seus sentimentos, pensamentos e ações. Assim, ao optar por realizar uma certa tarefa, o indivíduo também faz uma análise de suas habilidades e capacidades atuais, julgando se será ou não capaz de atingir seus objetivos na tomada de decisões para alcançá-los (PAJARES; OLAZ, 2008).

\section{O bullying escolar}

O bullying escolar é um problema que afeta crianças e adolescentes no mundo inteiro. De acordo com o relatório da UNESCO (2019), os maiores alvos de bullying são crianças e adolescentes em situações de vulnerabilidade, tendo como as principais causas a pobreza, status social associado a etnia, deficiências, diferenças linguísticas ou culturais, migração ou deslocamento, por serem órfãs ou que provém de famílias afetadas pelo HIV. No Brasil, a Pesquisa Nacional de Saúde do Escolar - PeNSE - apontou um crescimento de $35 \%$ desta violência nos ambientes escolares no período de 2009 a 2015 (MELLO et al., 2018), sendo que barreiras de comunicação e diferenças linguísticas representam fatores de risco para a vitimização por bullying. Em nível mundial, um conjunto de pesquisas confirmam esses aspectos ao identificar que os estudantes surdos e deficientes auditivos constituem um grupo alvo dessa prática nos espaços escolares (BAUMAN e 


\section{-†ı.}

Bullying, apoio social e senso de vida: relato de discentes surdas

Valéria Maria Azevedo Guimarães • Karine David Andrade Santos et al.

PERO, 2011; BROEKHOF, BOS, CAMODECA e RIEFFE, 2018; PINQUART e PFEIFFER, 2015; WEINER, DAY e GALVAN, 2013).

Lisboa, Braga e Ebert (2009) definem que o bullying é um fenômeno pelo qual uma criança ou um adolescente é sistematicamente exposta(o) a um conjunto de atos agressivos (diretos ou indiretos), que ocorrem sem motivação aparente, mas de forma intencional, protagonizados por um(a) ou mais agressor(es). Dentre os principais atos, Araújo e Silva (2011) comentam que existem várias formas de se praticar o bullying, a exemplo de agressões verbais, físicas, psicológicas e até virtual. Silva et al. (2017) apontam que, diferentemente, em relação aos agressores, a violência física é ressaltada, sugerindo que as formas verbais de violência não são identificadas como bullying. As consequências referentes ao bullying são variadas, e não são somente as vítimas que sofrem as consequências. Os agressores e as testemunhas também podem sofrer consequências tanto no âmbito emocional quanto na aprendizagem.

Vários são os efeitos produzidos por esses atos violentos e, em curto prazo, pode-se considerar que a vítima apresente insônia, reações psicossomáticas, pensamentos depreciativos e dificuldades na interação com demais colegas. Em longo prazo, pode manifestar dificuldade em se relacionar com outras pessoas, e as ações experienciadas podem influenciar no surgimento de quadros depressivos e, possivelmente, levar ao suicídio (CHAVES e SOUZA, 2018). Estudos têm associado bullying com solidão, ansiedade, insônia, tristeza, além de depressão e pensamentos suicidas. A literatura aponta a extensão do problema e as graves consequências para a saúde dos adolescentes, inclusive o estresse pós-traumático (MALTA et al., 2019). Estes acontecimentos também são encontrados na população surda, podendo ser ainda mais elevados e graves, por conta do isolamento escolar 


\section{_tı.}

Bullying, apoio social e senso de vida: relato de discentes surdas

Valéria Maria Azevedo Guimarães • Karine David Andrade Santos et al.

e das várias formas de manifestação do preconceito a que estão submetidos (ERNSEN, 2016).

\section{Libras e a comunidade surda}

Os surdos apresentam aspectos singulares em relação à cultura, ao uso da língua de sinais (LS), histórias, comportamentos, por exemplo, compondo assim, a comunidade surda (SMITH e RUSH, 2007). A Língua Brasileira de Sinais (Libras) é utilizada pela maior parte dos surdos (QUADROS e KARNOPP, 2004), e seu uso na comunidade surda possibilita a libertação dos estereótipos relacionados ao patológico, permitindo assim, desassociar a surdez da deficiência para ser compreendida como diferença linguística e cultural. Compreende-se que a LS é uma das formas que o sujeito possui para aprender, socializar e se desenvolver, sendo considerada um símbolo importante na identidade cultural do surdo. Entretanto, esta população também pode se identificar com outras culturas e identidades que pode envolver a língua portuguesa, por exemplo (GESSER, 2009).

Entretanto, a Libras ainda é desconhecida pela maior parte da sociedade e pelos próprios familiares de pessoas surdas. Aranda e Raaij (2015) salientam que, geralmente, os surdos nascem de pais ouvintes e seus genitores são inexperientes com a surdez e com a língua de sinais. Vale ressaltar que o sujeito inicia o processo de interação social, construção de crenças e desenvolve a maturidade no ambiente familiar. Além do amparo físico, emocional e social, a família orienta o sujeito sobre aspectos que podem trazer melhorias ou não para sua vida (NEGRELLI e MARCON, 2006). No entanto, du Feu (2014) e Glickman e Gulati (2003) ressaltam que a transmissão cultural dos surdos, usualmente, acontece por outros surdos e não pelos próprios pais, que frequentemente ouvem. Ademais, Fellinger et 


\section{_tı.}

Bullying, apoio social e senso de vida: relato de discentes surdas

Valéria Maria Azevedo Guimarães • Karine David Andrade Santos et al.

al (2009) e Kvam, Loeb e Tambs (2007) acrescentam que as barreiras existentes entre surdos e ouvintes e as experiências associadas ao estigma e discriminação têm o potencial de contribuir para a vulnerabilidade da população surda ao sofrimento mental. Além disso, Lacerda (2006) salienta que o desenvolvimento tardio da língua pode gerar consequências emocionais, sociais e cognitivas.

Isso pode ser percebido no processo histórico dos surdos quando Skliar (2016) aponta para o termo "ouvintismo" no intuito de salientar as práticas opressoras vivenciadas pela comunidade surda. O significado deste termo refere-se às representações dos ouvintes acerca da surdez e dos surdos, "o que impõe ao surdo a olhar-se e narrar-se como se fosse ouvinte" (p.15). Além disso, esse autor também cita o termo "oralismo" que é considerado "a forma institucionalizada do ouvintismo" (p.15). Desta forma, o ouvintismo, por ser uma ideologia dominante, se fortaleceu e teve a aceitação de profissionais da área da saúde e da educação e de familiares que foram em busca da ciência na tentativa de trazer a fala e a escuta para o surdo.

\section{Educação, apoio social e surdez}

A comunidade surda vem conquistando espaço na sociedade. Diante da importância da aprendizagem da língua de sinais, surge a oferta do ensino bilíngue nas escolas para os discentes surdos, em que a Libras é utilizada como primeira língua e o português escrito como segunda língua. De acordo com Nunes et al. (2015), o bilinguismo é defendido por entender que a língua de sinais faz parte da cultura surda e é por meio dela que os surdos são ouvidos e representados na sociedade. No estudo de Lederberg (1991), percebeu-se que infantes com perda auditiva e com conhecimento de língua 


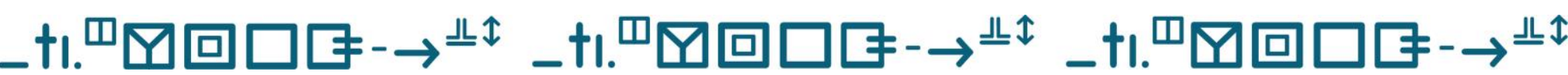

Bullying, apoio social e senso de vida: relato de discentes surdas

Valéria Maria Azevedo Guimarães • Karine David Andrade Santos et al.

tiveram maiores interações com colegas do que crianças com pouca capacidade de linguagem.

Apesar dos empecilhos da aprendizagem da língua de sinais, Whyte e Guiffrida (2008) apontam para os números de surdos, que ingressam na universidade, que vêm aumentando. O avanço da tecnologia e as conquistas dos direitos por meio de leis que exigem a presença de intérpretes em salas de aula, favoreceram o acesso dos discentes surdos a faculdades e universidades auditivas (planejadas para uma cultura ouvinte). Além disso, Brauer et al. (1998) acrescentam que estudantes universitários passam comumente por adaptações nesta nova etapa do desenvolvimento, como a ruptura de relações de apoio em casa, a construção de novas amizades e o gerenciamento das tarefas acadêmicas. No entanto, os surdos enfrentam desafios adicionais, pelo fato de geralmente necessitarem do intérprete para se comunicar com grande parte dos ouvintes que compõem o campus. Além disso, alguns discentes surdos podem ter dificuldade em manter um relacionamento próximo com docentes e funcionários da instituição, sem a presença do intérprete.

Os desafios na comunicação podem obstruir a convivência social que é importante para a formação da amizade. As relações com familiares, vizinhos e escola são suscetíveis de serem afetadas quando há pouca ou nenhuma habilidade de comunicação e desenvolvimento da linguagem, por serem aspectos relevantes para se manter uma relação de amizade (MOELLER, 2000).

Depois da família, frequentemente, o primeiro grupo significativo são os amigos. As amizades são favoráveis, pois são com elas que se desenvolvem variedades de habilidades sociais, como a cooperação, negociação, controle emocional e resolução de problemas (GAUVAIN, 2001). Laursen et al. (2007) e Ryan (2001) acrescentam que os amigos servem como 


\section{_tı.}

Bullying, apoio social e senso de vida: relato de discentes surdas

Valéria Maria Azevedo Guimarães • Karine David Andrade Santos et al.

modelos e também proporcionam companheirismo, segurança, apoio emocional, ajuda e orientação cognitiva. As amizades também podem ocasionar o aumento do senso de pertencimento e propósito das pessoas, a felicidade, dirimir o estresse e auxiliar com os desafios da vida (MILLEN, DORN e LUCKNER, 2019). Além do apoio social oferecido pela rede familiar e de amigos, outros recursos psicossociais são utilizados no enfrentamento de circunstâncias adversas ou estressantes, como o sentido de vida, um aspecto com potencial positivo no manejo de eventos desta natureza (HEINTZELMAN e KING, 2014).

\section{Sentido de vida e surdez}

Para a logoterapia ou a análise existencial, inaugurada por Frankl na década de 40 do século $X X$, o sentido de vida é a motivação básica do ser humano. Esta compreensão se fundamenta na nova dimensão humana concebida por este aporte teórico: a noética que movimenta o biológico e o psíquico na busca de sua realização existencial (FRANKL, 2011/2016). A autotranscedência, o autodistanciamento, a responsabilidade e a liberdade, componentes da dimensão noética, mobilizam comportamentos, atitudes e decisões que levam ao cumprimento das possibilidades de sentido da vida. A realização existencial em uma dada situação e de um indivíduo não é algo a ser dado, mas a ser encontrado pela própria pessoa com o auxílio da própria consciência que iluminará os valores e critérios para escolha das possibilidades de sentido (AQUINO, 2013; PEREIRA, 2013).

Estas oportunidades de sentido são materializadas pelo acionamento dos valores criativos, os valores vivenciais e os valores atitudinais. O primeiro se refere às criações intelectuais, de realização profissional, de objetivos, laborais e artísticas; o segundo é encontrado nas relações pessoais, na 


\section{_tı.}

Bullying, apoio social e senso de vida: relato de discentes surdas

Valéria Maria Azevedo Guimarães • Karine David Andrade Santos et al.

contemplação do belo e da natureza, e o terceiro emerge quando situações de sofrimento ou contextos irreversíveis são maiores que nossa capacidade humana de superá-los (FRANKL, 2016; XAUSA, 2013). Verifica-se que a realização existencial não é promovida internamente, mas direcionada para fora de si. Quando as pessoas refletem ativamente sobre o seu presente e seu futuro em diferentes áreas do seu viver (trabalho, família, lazer etc.), estabelecendo metas para cada aspecto fundamental da sua vida e em congruência com os seus valores, elas desenham um projeto de vida (SCHIPPERS e ZIEGLER, 2019). Diferentes estudos apontam que estar envolvido em um projeto de vida futuro está relacionado com o sucesso acadêmico e bem-estar estudantil (LOCKE e SCHIPPERS, 2018; SCHIPPERS et al., 2019).

No que concerne às experiências de dor e sofrimento, a literatura tem apontado que ter um propósito de vida é considerado um fator de proteção que minimiza os prejuízos decorrentes da vitimização, seja em jovens imersos em experiência comunitária (GURROLA-PENA et al., 2018), seja em sobreviventes de violência doméstica (BARA, CATHARINA e WARDANI, 2018). Ademais, o autodistanciamento e a autotranscedência são recursos disponíveis na dimensão noética humana que, mobilizados frente a vivências de dor e sofrimento, possibilitam ao homem se direciona a algo que não seja si mesmo e nem a dor experimentada pelo contexto irreversível, levando a construção de possibilidades de sentido nestas circunstâncias (XAUSA, 2013). Em relação aos surdos, um estudo de validação do questionário de sentido de vida para esta população revelou que este grupo buscava respostas de realização existencial frente a situações de sofrimento dentro da própria cultura surda (OLIVEIRA e AQUINO, 2014). 


\section{_tı.}

Bullying, apoio social e senso de vida: relato de discentes surdas

Valéria Maria Azevedo Guimarães • Karine David Andrade Santos et al.

\section{Método}

Participaram do estudo 07 alunas surdas, usuárias da Língua Brasileira de Sinais (Libras), matriculadas na graduação de Letras-Libras em uma instituição do ensino superior. Cabe salientar que o estudo foi aprovado pelo Comitê de Ética em Pesquisa Envolvendo Seres Humanos (CEP) 94220618.0.0000.5546, sob o número do parecer 2.897.758.

Foram estudados neste trabalho o bullying, o apoio social e o sentido de vida com universitárias surdas. Para isso, ocorreu na instituição em que as participantes estavam matriculadas uma entrevista semiestruturada em grupo, com a intermediação de dois profissionais tradutores e intérpretes de Libras-português. Portanto, as perguntas eram feitas pelas pesquisadoras em português e traduzidas pelos intérpretes para a Libras. Da mesma forma, as respostas das entrevistadas em Libras foram traduzidas para o português por estes profissionais. Constituíram a entrevista as seguintes indagações: $O$ que vem a sua mente com a palavra bullying?; Você se lembra de ter visto algum comportamento de violência na escola?; Quais são as histórias de bullying que você lembra na escola?; Com quem você contava na época da escola?; Como era o sentido de vida para você na época da escola e qual seu sentido de vida atual?; e Como você se imagina no futuro?.

Com o objetivo de conferência às informações e preservar a fidedignidade dos dados, as traduções tiveram seus áudios gravados e esses foram transcritos no software Microsoft Word pelas pesquisadoras posteriormente. Por conta de a tradução para o português ter sido realizada pelos intérpretes, foi necessária a gravação da voz desses profissionais com autorização.

O conteúdo textual obtido nas entrevistas foi organizado para a construção do corpus e submetido ao programa informatizado Interface de $R$ 


\section{_tı.}

Bullying, apoio social e senso de vida: relato de discentes surdas

Valéria Maria Azevedo Guimarães • Karine David Andrade Santos et al.

pour les Analyses Multidimensionnelles de Textes et de Questionnaires (IRAMUTEQ). Este é um método informático que propicia diferentes maneiras de análises textuais e resulta em uma organização compreensível e clara do material escrito coletado. Para este estudo, foi eleita a Classificação Hierárquica Descendente (CHD) que estrutura o vocabulário em classes, em um dendrograma, que evidencia as relações entre elas (CAMARGO e JUSTO, 2013).

\section{Resultados e discussão}

O corpus analisado por meio da Classificação Hierárquica Descendente (CHD) foi integrado por sete textos, fragmentados em 342 segmentos de texto (ST) e teve como aproveitamento de 266 segmentos de textos $(77,78 \%)$, o que é considerada uma retenção adequada para a classificação do material textual. Nesta análise, surgiram 829 formas (palavras) distintas e 783 palavras com uma ocorrência. Os STs são fragmentos de texto e o seu tamanho geralmente é de três linhas que são dimensionadas pelo próprio IRAMUTEQ, a depender do tamanho do corpus. Ademais, os segmentos de texto são classificados em função dos seus vocabulários e o agrupamento deles é repartido em função da frequência das formas reduzidas. Por meio das matrizes que cruzam segmentos de textos e palavras (por meio de repetições de testes do tipo $X^{2}$ ), emprega-se o método CHD e se alcança uma classificação estável (REINERT, 1990). Por meio da CHD se obtém classes de segmentos de textos que também apresentam vocabulários semelhantes entre si e vocabulários distintos dos segmentos de textos das outras classes. Mediante essas análises em matrizes, o IRAMUTEQ organiza a análise dos dados em um dendograma da CHD, que demonstra as relações entre as classes (CAMARGO e JUSTO, 2013). 


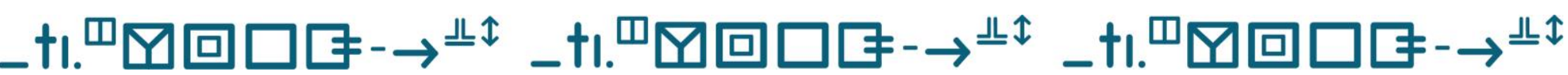

Bullying, apoio social e senso de vida: relato de discentes surdas

Valéria Maria Azevedo Guimarães • Karine David Andrade Santos et al.

Desta forma, as informações foram dispostas em quatro classes temáticas, divididas em dois subconjuntos e as dez primeiras palavras significativas foram escolhidas para ilustrar cada classe, como apresentado na Figura 1. Esta escolha foi feita pelo fato de o dendograma apresentar as palavras mais citadas e seu qui-quadrado - $X^{2}$ (para obter o contexto das palavras estatisticamente significativas), seguindo a mesma ordem exibida pelo programa IRAMUTEQ. A classe 4 foi a matriz das outras classes e está localizada no primeiro subgrupo, seguida da classe 3. As demais classes (classe 2 e classe 1) compõem o segundo subgrupo. Após essas análises e distribuições das classes, o próximo passo é a nomeação/categorização das classes encontradas baseadas nas temáticas trazidas em cada classe.

Figura 1. Dendograma da Classificação Hierárquica Descendente

\begin{tabular}{|c|c|c|c|c|c|c|c|c|c|c|c|}
\hline \multicolumn{3}{|c|}{$\begin{array}{c}\text { Classe 4 } \\
\text { Ponto de Partida } 20,68 \% \text { - 55ST } \\
\text { Apoio social e inclusão } \\
\text { acadêmica } \\
\end{array}$} & \multicolumn{3}{|c|}{$\begin{array}{c}\text { Classe 3 } \\
\text { Ponto de Partida 27,07\% - 72ST } \\
\text { Sentido de vida }\end{array}$} & \multicolumn{3}{|c|}{$\begin{array}{c}\text { Classe 2 } \\
\text { Ponto de Partida 24,44\% - } 65 \mathrm{ST} \\
\text { Relação familiar }\end{array}$} & \multicolumn{3}{|c|}{$\begin{array}{c}\text { Classe 1 } \\
\text { Ponto de Partida 27,82\% - 74ST } \\
\text { Bullying na escola }\end{array}$} \\
\hline Palavras & $f$ & $\mathrm{X}^{2}$ & Palavras & $f$ & $\mathrm{X}^{2}$ & Palavras & $f$ & $\mathbf{X}^{2}$ & Palavras & $f$ & $\mathrm{X}^{2}$ \\
\hline Aqui & 20 & 49,15 & Futuro & 29 & 60,34 & Pai & 24 & 59,96 & Escola & 17 & 38,73 \\
\hline Amigo & 11 & 23,99 & Vontade & 13 & 36,83 & Dizer & 33 & 39,2 & Ficar & 36 & 33,92 \\
\hline Graduação & 9 & 22,11 & Ajudar & 13 & 36,83 & Irmão & 12 & 30,05 & Bullying & 20 & 27,44 \\
\hline Vender & 5 & 19,55 & Vida & 21 & 33,9 & Casa & 15 & 29,94 & Gordo & 8 & 21,4 \\
\hline Diminuir & 5 & 19,55 & Aprender & 16 & 25,33 & Mãe & 26 & 28,04 & Porque & 29 & 18,17 \\
\hline Bom & 10 & 16,11 & Viajar & 9 & 25,1 & Perguntar & 8 & 25,51 & Chamar & 8 & 17,3 \\
\hline Forçar & 4 & 15,58 & Querer & 29 & 24,35 & $\begin{array}{c}\text { Namorar } \\
\text { Loja }\end{array}$ & 8 & $\begin{array}{l}22,33 \\
2096\end{array}$ & Lembrar & 9 & 16,66 \\
\hline Grupo & 4 & 15,58 & Mudar & 14 & 22,52 & & & 20,70 & Menos & 11 & 16,4 \\
\hline Confiar & 4 & 15,58 & Pensar & 18 & 21,96 & Esposo & 8 & 20,90 & Aluno & 6 & 15,93 \\
\hline Apoio & 4 & 15,58 & Sonho & 9 & 17,42 & Explicar & 5 & 15,76 & Ouvinte & 18 & 13,4 \\
\hline
\end{tabular}

Fonte: Os autores (2021).

Descrição da imagem: dendograma da CHD que demonstra as relações entre as classes. 


\section{_tı.}

Bullying, apoio social e senso de vida: relato de discentes surdas

Valéria Maria Azevedo Guimarães • Karine David Andrade Santos et al.

A análise do dendograma indicou que as participantes percebiam a graduação como um espaço de apoio social e emocional (classe 4 - "Apoio Social e Inclusão acadêmica"), buscavam sua realização existencial e superação das dificuldades por meio de metas e objetivos no futuro (classe 3 - "Sentido de Vida"). Em contrapartida, no passado, o ambiente familiar era caracterizado pela repressão (classe 2 - "Relação Familiar") e as participantes foram alvos de práticas de bullying no ambiente escolar (classe 1 - "Bullying na escola").

A classe 4 foi denominada de "Apoio Social e Inclusão acadêmica" e englobou 20,68\% dos segmentos do texto analisados. Os termos "aqui, amigo, graduação, vender, diminuir, bom, forçar, grupo, confiar e apoio" foram os mais citados, apontando para importância da inclusão acadêmica dos surdos e do encontro do apoio social nestes espaços. As palavras "aqui", "amigo", "graduação" e "apoio" abarcaram trechos que apontavam para o papel social e emocional promovido pela graduação:

Participante 1: Aqui na graduação quando eu imaginei essa questão da inclusão... Eu cheguei aqui bem dura e sem muita paciência. Depois eu fui compreendendo. Eu não tinha essa compreensão desse universo de inclusão.

Participante 2: A graduação estimula muito. A gente encontra mais apoio e eu encontrei aqui uma das minhas melhores amigas, né? Ela me orienta e me aconselha muito. Ela me traz muita motivação. Eu a amo.

Ramos (2015) afirma que a pessoa percebe a existência de suporte social quando ela sente que está em uma rede de interação composta por pessoas em que ela pode confiar e que a estimam. A inter-relação social, a vida em sociedade, permite a criação de apoio social nos diferentes ambientes onde o ser humano se encontra, e este apoio produz benefícios 


\section{_tı.}

Bullying, apoio social e senso de vida: relato de discentes surdas

Valéria Maria Azevedo Guimarães • Karine David Andrade Santos et al.

tanto para quem apoia, quanto para quem recebe o apoio (FONSECA e MOURA, 2008). O ambiente universitário pode ser um local propício, para que os indivíduos construam novas redes de apoio e as fortaleçam.

No caso da comunidade surda, o curso de Letras-Libras foi constituído com uma proposta voltada para o reconhecimento e valorização da cultura surda o que favorece a identificação dos surdos com seus pares e o protagonismo desta comunidade (QUADROS e STUMPF, 2009). A pesquisa de Matias e Martinelli (2017) considera que o apoio social entre os estudantes universitários é fundamental para a adaptação ao ensino superior. Ao investigar como se apresentava essa rede de apoio, foi verificada que a interação e o apoio afetivo foram mais indicados pelos participantes, aproximando-se dos resultados obtidos por Samssudin e Barros (2011). Esse suporte pode ser confirmado no fragmento a seguir:

Participante 1: Tem esse apoio, tem essa troca. Estamos sempre juntas, surdas/ouvintes. Há essa liberdade, mas antes quando eu cheguei aqui era bem fechada. Eu não queria ter essa relação, mas agora eu estou me sentindo bem melhor.

Esse apoio afetivo pode ser entendido como a capacidade que o ser humano tem de reconhecer os mais diversos sentimentos de um indivíduo diante de determinadas situações, compreendendo a necessidade e a intensidade do problema, sendo imprescindivel para a construção das relações sociais. Cechet (2013) menciona que o estreitamento dos laços entre os estudantes permite o compartilhamento de expectativas, interesses e problemas, facilitando a adaptação. O estudo de Zampar (2015) sobre a integração na universidade identificou o apoio dos colegas como fator determinante para facilitar a integração e/ou permanência na universidade 


\section{-†ı.}

Bullying, apoio social e senso de vida: relato de discentes surdas

Valéria Maria Azevedo Guimarães • Karine David Andrade Santos et al.

por todos os participantes, enquanto que a pesquisa de Santos, Oliveira e Dias (2015) considera que a troca de experiências com colegas pode ser fundamental para dar sentido ao percurso do discente na faculdade, de modo que ele se sinta emocionalmente apoiado e menos ansioso. Esses vínculos podem exercer influência significativa na vida desses sujeitos, como verificado na frase a seguir:

Participante 3: Depois que eu vim aqui para a graduação, eu vi o interesse das pessoas, a interação. Eu fiquei vendo as pessoas se desenvolvendo. Eu gostei muito daqui da graduação, entendeu? Eu queria vir para a graduação mesmo.

O apoio social e emocional promovido pelo ambiente universitário em questão foi permitido pela efetivação de políticas de inclusão (MARTINS e NAPOLITANO, 2017), a saber: Programa Incluir 13, Decreto $n^{\circ} 3.298 / 1999$, Portaria n 3.284/2003, Circular no 277/1996, Decreto $n^{\circ} 5.626 / 2005$, Lei $n^{\circ}$ 12.319/2010, Atendimento Diferenciado para o ENEM (INEP, 2012), Lei $n^{\circ}$ 13.146/2015 - Lei Brasileira de Inclusão (BRASIL, 2015). Essas políticas passam a garantir às pessoas surdas, o ingresso e permanência na universidade. Tratam-se de práticas sociais aplicadas no trabalho, na arquitetura, no lazer, na educação, na cultura, mas, principalmente, na atitude e no perceber das coisas, de si e do outrem (CAMARGO, 2017).

Apesar dos avanços em relação às leis que garantam a acessibilidade dos deficientes no ensino superior, há uma distância entre teoria e prática (SANTANA, 2016), tendo em vista que as políticas direcionadas às pessoas com deficiência são representadas em seus desdobramentos no que diz respeito à acessibilidade em todos os seus aspectos. Para se pensar no ensino das pessoas surdas, é preciso levar em consideração os direitos linguísticos (MESQUITA, 2018), em busca de uma educação bilíngue que 


\section{_tı.}

Bullying, apoio social e senso de vida: relato de discentes surdas

Valéria Maria Azevedo Guimarães • Karine David Andrade Santos et al.

valide e valorize as singularidades linguísticas dos surdos (SARTORI, 2019). Olhar o outro e, também, a si mesmo dentro do processo de inclusão pode ser a oportunidade de mudança de práticas excludentes e o início de ações inclusivas efetivas, como ilustrado no segmento abaixo:

Participante 1: Eu não queria ter essa relação porque eu achava que elas não iam se esforçar, não iam conseguir sinalizar. Agora eu vejo elas conversando, sinalizando, se esforçando. Um apoio, suporte entre nós mesmas.

Torna-se salutar que sejam empreendidas políticas sociais efetivas, que resolvam os problemas e todos os seus efeitos, até mesmo, os de ordem psicológica, que afetam as pessoas envolvidas (ALVES, LEÃO e AGAPITO, 2017). É imprescindível que sejam elaboradas políticas públicas compatíveis com a realidade dos sujeitos surdos para que, de fato, eles integrem a sociedade e possam se desenvolver social, linguística e educacionalmente. Observou-se, durante a análise desta classe, que a efetivação de práticas inclusivas e construção das relações sociais são primordiais para o apoio emocional das universitárias pesquisadas.

A classe 3 foi denominada de "Sentido de Vida" e concebeu 27,07\% dos segmentos do texto analisados. As palavras citadas com maior frequência pelos participantes foram "futuro, vontade, ajudar, vida, aprender, viajar, querer, mudar, pensar e sonho", sendo usadas para expressar o caminho que as estudantes seguiram para superar as frustrações e, ao mesmo tempo, construir as mais variadas formas de buscar o sentido da vida nos contextos adversos.

Uma das maiores dificuldades vivenciadas pelas participantes foi a ausência de interação entre indivíduos ou grupos sociais, principalmente das relações que se estabeleciam entre pessoas surdas e ouvintes. As 


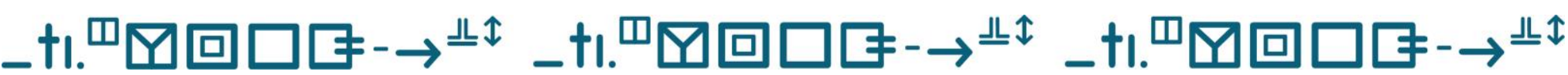

Bullying, apoio social e senso de vida: relato de discentes surdas

Valéria Maria Azevedo Guimarães • Karine David Andrade Santos et al.

dificuldades comunicativas, que se iniciavam já no ambiente familiar, posteriormente se estendiam ao resto da sociedade ouvinte, como já relatada em outras pesquisas (LOPES e LEITE, 2011). A presença de sentido surgia quando a barreira comunicacional impossibilitava as relações sociais. Estas representações podem ser ilustradas nas frases a seguir:

Participante 02: Antes eu tinha alguns traumas com ouvintes, mas depois foi melhorando, fui compreendendo sobre essa realidade dos ouvintes. Através do exercício, do dia a dia, da paciência, do contato, da aprendizagem foi se criando uma nova relação e fui avançando.

Esta superação foi promovida pelo exercício dos valores atitudinais, que são o modo como o homem se comporta perante as situações que geram sofrimento e não podem ser mudadas (FRANKL, 2015; SILVEIRA e GRANDIM, 2015). Ressignificar sua existência é crescer para além de si e renascer maior do que antes. Na medida em que o indivíduo realiza valores, salva as possibilidades no passado, podendo recuperá-las no presente, para transformá-las no futuro, a partir da consecução de sua tarefa pessoal (CORRÊA e RODRIGUES, 2013; FRANKL, 2018). As experiências positivas das ações praticadas diariamente proporcionavam boas expectativas para 0 futuro, como observado na seguinte passagem:

Participante 01: Comecei a aprender mais, conhecer pessoas, ouvintes também. Várias pessoas que me orientam e me aconselham. Foi bem melhor. Eu desenvolvi bastante, aprendi, estou conseguindo abrir mais a minha mente, entendeu? 


\section{_tı.}

Bullying, apoio social e senso de vida: relato de discentes surdas

Valéria Maria Azevedo Guimarães • Karine David Andrade Santos et al.

O sofrimento faz parte do modo de vida do ser humano e, cabe ao homem aceitá-lo e procurar integrá-lo no desenvolvimento de sua história pessoal, ou rejeitá-lo através da negação, da indiferença ou do relativismo existencial (GUIMARÃES, 2012). Esta ocorrência pode ser observada no relato a seguir:

Participante 01: Eu entendo minha vida agora, mas o futuro ainda não entendo. Eu acredito que ainda vai melhorar, vai mudar minha vida no futuro.

Verificou-se também que o público pesquisado buscava a sua realização existencial, por meio de sonhos, metas e realizações no futuro, o que atesta o acionamento dos valores criativos. Este é entendido, pela teoria frankliana, como a capacidade de dar algo ao mundo, oferecer algo por meio de criações, ou seja, um trabalho (CORRÊA e RODRIGUES, 2013). Esta satisfação do ser humano em se sentir útil, por meio de trabalho ou atividades, mesmo que seja uma idealização desta atividade no futuro, pode ser observada em estudos realizados com outras populações (WARD e KING, 2017; ZHANG et al., 2019). O trecho abaixo ilustra a ideia apresentada:

Participante 02: Ser uma pesquisadora, apropriar-me disso tudo para poder me desenvolver. Quero fazer universidade, doutorado. Então, sempre estou pesquisando, buscando informações nesse contexto em geral para aumentar o meu conhecimento. A minha vida poder mudar nesse sentido.

Em contrapartida, as experiências de sofrimento não ressignificadas pelas participantes abriram caminhos para o vazio existencial, que se caracteriza pela perda de sentido na vida e pela vivência do autocentramento (XAUSA, 2013). O fastio causado pelos acontecimentos desagradáveis fez 


\section{_tı.}

Bullying, apoio social e senso de vida: relato de discentes surdas

Valéria Maria Azevedo Guimarães • Karine David Andrade Santos et al.

com que as participantes não mobilizassem a liberdade e a responsabilidade, aspectos fundantes da dimensão humana segundo a perspectiva logoterapêutica (FRANKL, 2018). A importância do sentido de vida pode ser corroborada por estudos que apontam como a presença deste aspecto é um fator de proteção para os efeitos nocivos oriundos de experiências de violência como assédio (LIU et al., 2020) e bullying (HENRY et al., 2014), episódios vivenciados pelas participantes, como relatado em outras classes. Segue fragmento que ilustra o vazio existencial vivenciado por uma das participantes:

Participante 01: Você era tão feliz e agora não é mais? Realmente, as pessoas não me respeitam, não querem que eu aprenda mais e eu preciso ter essas informações.

Nesta classe, observou-se que um dos maiores obstáculos para o sujeito surdo foi a barreira comunicacional que trazia, nas diferentes interações, cenários de sofrimento e violência, cujo enfrentamento pelas participantes era facilitado pelo encontro do sentido de vida nestas circunstâncias. A perspectiva de uma atividade no futuro também figurou como uma via de acesso para um sentido de suas existências. Por outro lado, o sofrimento vivenciado pelas estudantes surdas nos contextos difíceis era potencializado, quando elas não encontravam a realização existencial nestas circunstâncias.

A classe 2, por sua vez, foi denominada de "Relação Familiar" e representou 24,44\% dos segmentos do texto analisados. Esta classe envolveu temáticas acerca das relações familiares e amorosas, englobando conteúdos sobre os direitos e a busca da independência. Os vocábulos "pai, dizer, irmão, casa, mãe, perguntar, namorar, loja, esposo e explicar" 


\section{_tı.}

Bullying, apoio social e senso de vida: relato de discentes surdas

Valéria Maria Azevedo Guimarães • Karine David Andrade Santos et al.

representam a maneira como os pais se comportavam diante de seus filhos surdos. Além disso, havia comparações entre os irmãos ouvintes e surdos, em busca de direitos igualitários.

Participante 04: Conversei com meu pai e avisei que iria me mudar, alugar uma casa e viver minha vida. Meu pai mesmo assim não deixou. Eu com 20 anos, fui embora. Meu pai foi me procurar, saiu mostrando a minha foto e me encontrou. Apanhei. Era apanhando e eu correndo.

Por meio da fala supracitada, pode-se perceber que não havia uma comunicação efetiva no meio familiar em um período de desenvolvimento da discente, a adolescência, caracterizado pela intensidade das emoções, busca do autoconhecimento, a procura por grupos e o distanciamento familiar (WAGNER et al., 2002). Para lidar com esta fase da vida, os pais da entrevistada adotavam práticas coercitivas que, em geral, são aversivas e provocam emoções negativas como medo e ansiedade, predispondo o indivíduo a fugir, retrucar e/ou agredir seu agressor/punidor (PATIAS, SIQUEIRA e DIAS, 2012). Nota-se que a violência nas relações traz mais uma barreira adicional na comunicação entre os surdos e os familiares e, por consequência, estes não conseguem acessar as angústias existentes dos filhos, o que contribui para o distanciamento entre os sujeitos surdos e seus familiares (BATISTA e REIS, 2011), ocasionando um espaço propício para malentendidos nas relações.

Participante 06: Meu pai não me entendia. Só queria fazer do jeito dele. Ele não deixava sair com minha prima. Ele não me deixava fazer nada. Minha mãe explicava para ele que eu precisava ter experiência de sair sozinha. Eu ficava angustiada com tudo isso. 


\section{-†ı.}

Bullying, apoio social e senso de vida: relato de discentes surdas

Valéria Maria Azevedo Guimarães • Karine David Andrade Santos et al.

Dalcin (2009) salienta que os mal-entendidos provocam angústia no surdo, porque a falta de informação ou redução desta e a ausência de clareza dos motivos/causas de solicitações e/ou ordens recebidas faz com que desconheça de fato situações sobre si e sobre o mundo. Silva e Bastos (2013) abordam a insegurança dos pais ouvintes em relação à condição linguística de seus filhos surdos e afirmam que isso pode ocasionar, na maioria dos casos, as atitudes superprotetoras. A falta de uma língua acarreta sérias dificuldades de convivência e nos surdos essa é uma condição frequente, ocasionada pelo diagnóstico tardio e consequentemente pelo fato de algumas crianças surdas se desenvolverem sem serem expostas a qualquer língua de forma acessível. Desta forma, a falta desta exposição pode prejudicar no desenvolvimento cognitivo e afetivo do sujeito. Augusto e Chacon (2011) acrescentam que os extremos (superproteger e o abandono) podem trazer dificuldades para a prole e familiares.

As situações citadas são frequentes na vida dos sujeitos surdos e, em muitos casos, acabam gerando uma proteção excessiva por parte dos familiares, principalmente quando se trata do sexo feminino. A mulher surda gera nos familiares insegurança e receio por acreditarem que a surdez torna a mulher um ser desprotegido e dependente de seus familiares em todas as etapas da vida. As crenças limitadoras impossibilitam os pais de enxergarem as aptidões de suas filhas surdas, sendo estas capazes de conquistar seus objetivos de vida como qualquer pessoa, a exemplo, trabalhar, morar fora, independência financeira e, até, namorar. Os pais não conhecem a surdez do ponto de vista dos próprios surdos, assim como podem não conhecer pessoas surdas adultas, não saber como elas agem, como é o seu dia a dia, como se comunicam, como pensam e sentem (STELLING et al., 2014). Verificou-se, nesta classe, que as relações familiares das discentes surdas 


\section{_tı.}

Bullying, apoio social e senso de vida: relato de discentes surdas

Valéria Maria Azevedo Guimarães • Karine David Andrade Santos et al.

eram permeadas por atitudes coercitivas e de proteção excessiva que afetaram a qualidade da comunicação entre os membros familiares e o desenvolvimento emocional das participantes.

Por fim, a classe 1 foi denominada "Bullying na escola" e representou $27,82 \%$ dos segmentos do texto analisados. Nesta classe, as palavras "escola, ficar, bullying, gordo, porque, chamar, lembrar, menos, aluno e ouvinte" expressaram a ocorrência, localidade e o tipo do bullying sofrido pelos participantes durante a infância e/ou adolescência e as respectivas consequências dessa prática. Os trechos a seguir são ilustrativos da presença destas ações direcionados aos surdos que ocorriam, de maneira predominante, nos ambientes escolares e em outros locais, como no trabalho e na rua:

Participante 02: Na escola tinha bullying. As pessoas acham normal as brincadeiras. Ficavam me provocando, dizendo que eu tinha nariz de bruxa.

Participante 03: Em relação aos surdos que estão em casa, na rua, tem várias pessoas que sofrem bullying, apanham. Pode ser em qualquer lugar, na escola, na rua, no trabalho.

Essas práticas abusivas passaram a ser denominadas de bullying, conceituada por Souza (2013), como atitudes violentas praticadas por um ou mais indivíduos contra terceiros, cuja finalidade é apenas maltratar e intimidar. Estudos em diferentes países têm apontado que aparência física, comportamentos individuais, nível de desempenho na escola, aspectos religiosos, questões de gênero, orientação sexual, afrodescendentes e deficiências são os principais motivadores para a ocorrência da prática do bullying nos espaços escolares (OLIVEIRA et al, 2015). 


\section{_tı.}

Bullying, apoio social e senso de vida: relato de discentes surdas

Valéria Maria Azevedo Guimarães • Karine David Andrade Santos et al.

Os discentes com deficiências são frequentemente alvos dos agressores (FLYNT e MORTON, 2004; NORWICH e KELLY, 2004; WHITNEY, SMITH e THOMPSON, 1994). Segundo Kent (2003) e Sheridan (2001), a população surda e com deficiência auditiva relataram casos de terem sido provocados por seus colegas ouvintes na escola ou em seus bairros o que corrobora os achados desta pesquisa. De acordo com Souza (2019), no ambiente escolar, esse tipo de violência inicia com perseguições ou intimidações contra um indivíduo, por parte de terceiros, sem motivos específicos. Tais comportamentos visam prejudicar, ferir, desestabilizar o sujeito e estabelecer uma relação dominante-dominado entre o agressor e a pessoa agredida. As principais agressões que caracterizam ações de bullying podem ser tanto de ordem física quanto psicológica. As ações violentas mais frequentes são bater, chutar, pontapear, agredir com armas, ameaçar, insultar, debochar, apelidar, humilhar, excluir, extorquir e ser indiferente com a vítima (MARCOLINO et al., 2018). As participantes eram alvos de práticas de insulto e provocações, como destacado no segmento a seguir.

Participante 04: No passado, na escola com ouvinte, misturado, era normal a gente conversava. Mas, eu era surda e alguns ficavam me provocando, dizendo que eu era burra, que não sabia português, que eu não sabia escrever as frases e eu ficava com aquele sentimento ruim.

A exemplo do relato supracitado, foi possível identificar nos dados desta pesquisa que a não aceitação da diferença por parte do agressor pode ocasionar na vítima, dificuldade social, educacional e emocional o que corrobora o estudo de Ernsen (2016) sobre o bullying e surdez. O efeito produzido pelo bullying é observado no fragmento a seguir. 


\section{_tı.}

Bullying, apoio social e senso de vida: relato de discentes surdas

Valéria Maria Azevedo Guimarães • Karine David Andrade Santos et al.

Participante 03: Na escola tinha bullying. Dependia da consciência de cada um. A mãe precisava ensinar eles a terem respeito. Às vezes, eu ficava deprimida com várias coisas.

Os atos de violência praticados de maneira constante e de forma proposital podem ocasionar uma série de consequências sociais que, segundo Sampaio et al. (2015), apresentam ocorrência imediata e impactam negativamente na qualidade de vida e de escolarização dos estudantes. A literatura tem relatado que os sintomas depressivos nos alvos de bullying são causados pela modalidade verbal deste tipo de violência (COUTINHO et al., 2016; FORLIM, STELKO-PEREIRA e WILLIAMS, 2014; GROLLI, WAGNER e DALBOSCO, 2017). Sentimentos de rejeição, medo, raiva e ansiedade em relação aos seus pares estão presentes nos sujeitos alvos de bullying (COUTINHO et. al., 2016). Dentre as inúmeras causas que podem motivar agressões contra estudantes com deficiência, especificamente pessoas surdas, está a barreira linguística. É sabido que a condição linguística de um indivíduo não é o único limitador da causa dos atos violentos e, muito menos, do agressor/vítima dessas práticas abusivas. Ernsen (2016) pontua que o bullying acontece não só entre um agressor ouvinte e uma vítima surda, como também entre um agressor surdo sinalizante e uma vítima surda não usuária da língua de sinais e/ou oralizada e/ou que utiliza implante coclear.

Nesta classe, constatou-se que os ambientes escolares são os locais predominantes de ocorrência das práticas de bullying do tipo verbal direcionadas às estudantes surdas, sendo que as ações violentas tinham, como fundo, a diferença linguística. No contexto de violência, as estudantes vivenciavam adversidades sociais, de aprendizagem e emocionais que contribuíam para a manifestação de sintomas depressivos em alguns casos. 


\section{_tı.}

Bullying, apoio social e senso de vida: relato de discentes surdas

Valéria Maria Azevedo Guimarães • Karine David Andrade Santos et al.

\section{Conclusão}

Por meio dos dados analisados no presente estudo, verificou-se que o espaço universitário que as participantes da pesquisa estão inseridas constitui hoje, para elas, uma fonte de apoio social e emocional, apontandose para a importância da efetivação de políticas públicas de inclusão neste nível de ensino. É válido destacar que as leis e os decretos relacionados à inclusão e acessibilidade e as políticas educacionais voltados para a população surda, citados no decorrer do manuscrito, beneficiam a educação dos surdos em todos os níveis de instrução (da educação básica ao ensino superior), possibilitando aproximações e experiências com diferentes públicos. Outro aspecto que pode ser mencionado são as narrativas das entrevistadas referentes às suas experiências de sofrimento provocadas pelos obstáculos na comunicação, pois elas buscavam diferentes caminhos para acessar a realização existencial ou sentido de vida nestas circunstâncias adversas.

Na infância e adolescência das participantes, observou-se que a relação familiar era caracterizada por situações de conflitos com os pais, nos movimentos de busca de independência e de exercício de direitos. Estas tensões eram oriundas de um contexto de superproteção familiar, principalmente, pelo fato de serem mulheres. No ambiente escolar, elas eram alvos de práticas de bullying, como insultos e provocações, que causavam prejuízos sociais e emocionais na interação das pesquisadas com os seus pares nestes espaços.

Os resultados apontaram para o papel fundamental exercido pelo apoio social e emocional ofertado por amigos, colegas e pelo uso da língua de sinais em um espaço universitário, constituindo uma experiência acadêmica que se contrapõe às práticas de bullying experimentadas pelas 


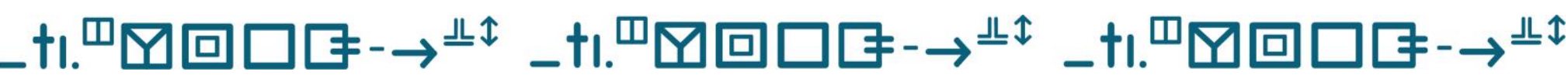

Bullying, apoio social e senso de vida: relato de discentes surdas

Valéria Maria Azevedo Guimarães • Karine David Andrade Santos et al.

pesquisadas no ambiente escolar. Constatou-se que, quando os espaços educacionais se baseiam nas políticas de inclusão escolar e promovem adaptações baseadas na cultura surda, podem fornecer aos estudantes surdos experiências bem-sucedidas e estas apresentam um potencial de redesenhar histórias de vida marcadas pela dor e frustração oriundas de espaços não inclusivos, de relações familiares disfuncionais e de barreiras impostas por uma sociedade que geralmente se fundamenta nos ideais do ouvintismo e do oralismo. Outra fonte de ressignificação das experiências de vida era transcender estes contextos, por meio do encontro do sentido nestas situações, e ter metas e sonhos inscritos em uma perspectiva de projeto de vida no futuro.

Sugere-se que sejam realizados estudos longitudinais para aferir o impacto da oferta de apoio social e emocional e da presença de sentido de vida nos indicadores de saúde mental de surdos. Também é oportuno avaliar quais aspectos dos espaços universitários que influenciam a percepção positiva de apoio social destas estudantes. Em contrapartida, as experiências de bullying vivenciadas pelas participantes apontam para a necessidade de repensar o tipo de educação inclusiva ofertada pelas escolas, e as modalidades de intervenção de combate a esta prática violenta nestes espaços. Recomenda-se que, antes de qualquer intervenção de combate ao bullying, estas estudantes sejam efetivamente acolhidas pelos espaços escolares, por meio da implementação de melhorias e adaptações de sua estrutura física, de recursos educacionais, dos modelos de ensinoaprendizagem e demais ferramentas necessárias e específicas para cada especificidade.

Este estudo apresenta algumas limitações que impedem a abrangência dos resultados para a comunidade surda. A primeira se refere à quantidade limitada de estudantes disponíveis para participar desta 


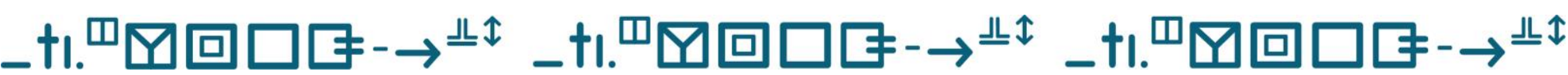

Bullying, apoio social e senso de vida: relato de discentes surdas

Valéria Maria Azevedo Guimarães • Karine David Andrade Santos et al.

investigação. A segunda se refere à amostra ser constituída apenas por estudantes do sexo feminino, impossibilitando avaliar se há diferenças nas experiências familiares, escolares, de apoio nos espaços universitários e de sentido de vida entre surdos e surdas.

\section{Referências}

ALVES, L. F.; LEÃO, M. F.; AGAPITO, F. M. Políticas Públicas voltadas para a inclusão social dos surdos, Revista Destaques Acadêmicos, v. 9, n. 2, p. 149162, 2017.

AQUINO, T. A. A. Logoterapia e Análise Existencial: uma introdução ao pensamento de Viktor Frankl. São Paulo: Paulus, 2013.

ARANDA, B.; RAAIJ, I. S. V. Mental health services for deaf people: treatment advances, opportunities and challenges. Washington: Gallaudet University Press, 2015.

ARAÚJO, C. P. S.; SILVA, L. R. Bullying na escola: essa brincadeira não tem graça. In: V Colóquio Internacional Educação e Contemporaneidade, 2011, São Cristóvão. Anais, São Cristóvão: Universidade Federal de Sergipe, p. 1-16, 2011.

AUGUSTO, M. A. P. C.; CHACON, M. C. M. Diferentes enfoques das relações familiares: superproteção e abandono. In: VII Encontro da Associação Brasileira de Pesquisadores em Educação Especial, Londrina. Anais, Londrina: p. 1316-1327, 2011.

BARA, I. M. S. B.; CATHARINA, N. H.; WARDANI I. Y. Meaning of Life of Adolescent Survivors of Domestic Violence (DV). Advanced Science Letters, v. 24, n. 9, p. 6735-6738, 2018.

BATISTA, T. P. M.; REIS, J. G. A família de estudantes surdos: e a importância da comunicação em libras para processo de aprendizagem. In: VII Encontro 


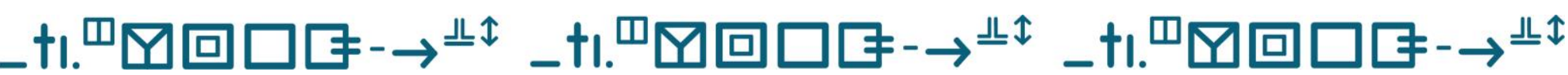

Bullying, apoio social e senso de vida: relato de discentes surdas

Valéria Maria Azevedo Guimarães • Karine David Andrade Santos et al.

\section{da Associação Brasileira de Pesquisadores em Educação Especial,}

Londrina. Anais, Londrina, p. 1201-1213, 2011.

BAUMAN, S.; PERO, H. Bullying and cyberbullying among deaf students and their hearing peers: An exploratory study. Journal of deaf studies and deaf education, 16(2), 236-253, 2011.

BRASIL. 0 atendimento diferenciado no ENEM. Instituto Nacional de Estudos e Pesquisas Educacionais Anísio Teixeira (INEP), 2012. Disponível em: <http://download.inep.gov.br/educacao_basica/enem/nota_tecnica/2012/at endimento_diferenciado_enem_2012.pdf>

BRASIL. Lei no 13.146, de 6 de julho de 2015 - Lei Brasileira de Inclusão das pessoas com deficiência. Disponível em: <http://www.planalto.gov.br/ccivil 03/ ato2015-2018/2015/lei/l13146.htm>. Acesso em:

BRAUER, B. A.; BRADEN, J. P.; POLLARD, R. Q.; HARDY-BRAZ, S. T. Deaf and hard of hearing people. In: SANDOVAL, J. H.; FRISBY, C. L.; GEISINGER, K. F.; J. R. GRENIER; SCHEUNEMAN, J. D. Test interpretation and diversity: Achieving equity in assessment. Washington: American Psychological Association, p. 297-315, 1998.

BROEKHOF, E., BOS, M. G., CAMODECA, M.; RIEFFE, C. Longitudinal associations between bullying and emotions in deaf and hard of hearing adolescents. The Journal of Deaf Studies and Deaf Education, 23(1), 17-27, 2018.

CAMARGO, B. V.; JUSTO, A. M. IRAMUTEQ: Um software gratuito para análise de dados textuais. Temas em Psicologia, 21(2), 513-518, 2013.

CAMARGO, E. P. Inclusão social, educação inclusiva e educação especial: enlaces e desenlaces. Ciência e Educação, v. 33, n. 1, 2017.

CECHET, A. G. S. 0 ingresso na universidade pública: análise dos sentidos atribuídos por um grupo de estudantes atendidos pela assistência estudantil. 


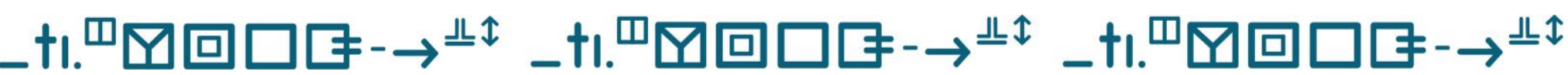

Bullying, apoio social e senso de vida: relato de discentes surdas

Valéria Maria Azevedo Guimarães • Karine David Andrade Santos et al.

f. 89. Dissertação (Mestrado em Psicologia) - Setor de Ciências Humanas, Universidade Federal do Paraná, Curitiba, 2013.

CHAVES, D. R. L.; SOUZA, M. R. Bullying e preconceito: a atualidade da barbárie. Rev. Bras. Educ., v. 23, p. 1-17, 2018.

CORREAA, D. A.; RODRIGUES, C. M. D. Finitude e sentido de vida: do torpor à tarefa. Revista da Associação Brasileira de Logoterapia e Análise Existencial - Logos \& existência, v. 2, n. 1, p. 37-46, 2013.

COUTINHO, M. P. L.; PINTO, A. V. L.; CAVALCANTE, J. G.; ARAUJO, L. S.; COUTINHO, M. L. Relação entre depressão e qualidade de vida de adolescentes no contexto escolar. Psicologia, Saúde e Doença, v. 17, n. 3, p. 338-351, 2016.

DALCIN, G. Psicologia da educação de surdos. Universidade Federal de Santa Catarina, Curso de Licenciatura em Letras-Libras na Modalidade a Distância, Florianópolis, 2009.

DU FEU, M. Deafness: The Facts. In M., du Feu \& C., Chovaz (Orgs.). Mental Health and Deafness. Oxford University Press, 2014.

ERNSEN, B. P. Bullying e surdez no contexto escolar. Dissertação (Mestrado em Educação) - Programa de Pós-Graduação em Educação, na linha de pesquisa de Cognição, Aprendizagem e Desenvolvimento Humano, Universidade Federal do Paraná, Curitiba, 2016.

FELLINGER， J.; HOLZINGER，D.; SATTEL，H.; LAUCHT， M.; GOLDBERG，D. Correlates of mental health disorders among children with hearing impairments. Dev Med Child Neurol, v. 51, p. 635-4, 2009.

FLYNT, S. W.; MORTON, R. C. Bullying and children with disabilities. Journal of Instructional Psychology, v. 31, n. 4, 330-333, 2004.

FONSECA, I. S. S.; MOURA, S. B. Apoio social, saúde e trabalho: uma breve revisão. Psicol. Am. Lat., n. 15, 2008. 


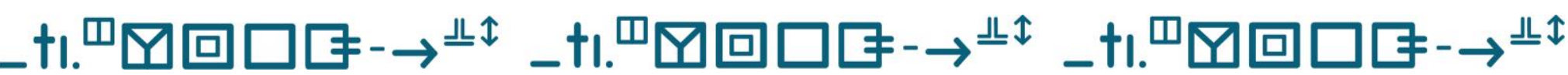

Bullying, apoio social e senso de vida: relato de discentes surdas

Valéria Maria Azevedo Guimarães • Karine David Andrade Santos et al.

FORLIM, B. G.; STELKO-PEREIRA, A. C.; WILLIAMS, L. C. A. Relação entre bullying e sintomas depressivos em estudantes do ensino fundamental. Estudos de Psicologia, v. 31, n. 3, p. 367-375, 2014.

FRANKL, V. E. A vontade de sentido: fundamentos e aplicações da logoterapia. São Paulo: É Realizações, 2011.

FRANKL, V. E. O sofrimento de uma vida sem sentido. São Paulo: Paulus, 2015.

FRANKL, V. E. Psicoterapia e sentido de vida: fundamentos da logoterapia e análise existencial. São Paulo: Quadrante, 2016.

FRANKL, V. E. Psicoterapia para todos. Petropólis: Vozes, 2018.

GAUVAIN, M. The social context of cognitive development. New York: Guilford Press, 2001.

GESSER, A. LIBRAS? Que língua é essa?: Crenças e preconceitos em torno da língua de sinais e da realidade surda. São Paulo: Parábola, 2009.

GLICKMAN, N. S.; GULATI, S. Mental health care of deaf people: a culturally affirmative approach. Lawrence Erlbaum Associates: London, 2003.

GUIMARÃES, F. F. O sentido do sofrimento humano. Revista do Hospital Universitário Pedro Ernesto, v. 11, n. 2, p. 70-76, 2012.

GURROLA-PENA, G. M., BALCAZAR-NAVA, P.; VILLAR, O. E.; LUIS, A. G.; ALMANZA-AVENDANO, M. A. Protective Factors for the Development of Psycho-pathological Symptoms in Young Victims of Community Violence. International Journal of Emergency Mental Health and Human Resilience, v. 20, n. 1, p 1-9, 2018.

GROLLI, V.; WAGNER, M. F.; DALBOSCO, S. N. P. Sintomas Depressivos e de ansiedade em adolescentes do ensino médio. Revista de Psicologia IMED, v. 9, n. 1, p. 87-103, 2017.

HENRY, K. L.; LOVEGROVE, P. J.; STEGER, M. F.; CHEN, P. Y.; CIGULAROV, K. P.; TOMAZIC, R. G. The potential role of meaning in life in the relationship 


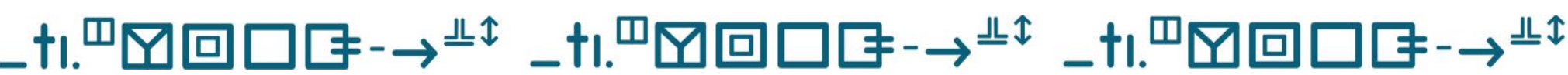

Bullying, apoio social e senso de vida: relato de discentes surdas

Valéria Maria Azevedo Guimarães • Karine David Andrade Santos et al.

between bullying victimization and suicidal ideation. Journal of youth and adolescence, v. 43, n. 2, p. 221-232, 2014.

HEINTZELMAN, S. J.; KING, L. A. Life is pretty meaningful. American Psychologist, 69, 561-574, 2014.

KENT, B. A. Identity issues for hard-ofhearing adolescents aged 11, 13, and 15 in mainstream settings. Journal of Deaf Studies and Deaf Education, v. 8, n. 3, 315-324, 2003.

KVAM, M.; LOEB, M.; TAMBS, K. Mental health in deaf adults: symptoms of anxiety and depression among hearing and deaf individuals. J Deaf Stud Deaf Educ., v. 12, n. 1, p. 1-7, 2007.

LACERDA, C. B. F. A inclusão escolar de alunos surdos: o que dizem alunos, professores e intérpretes sobre esta experiência. Cad. Cedes, Campinas, v. 26, n. 69, p. 163-184, 2006.

LAURSEN, B.; BUKOWSKI, W. M.; AUNOLA, K.; NURMI, J. E. Friendship moderates prospective associations between social isolation and adjustment problems in young children. Child Development, v. 78, n. 4, p. 1395-1404, 2007.

LEDERBERG, A. R. Social interaction among deaf preschoolers: The effects of language ability and age. American Annals of the Deaf, v. 136, n. 1, p. 5359, 1991.

LISBOA, C.; BRAGA, L. L.; EBERT, G. O fenômeno bullying ou vitimização entre pares na atualidade: definições, formas de manifestação e possibilidade de intervenção. Contextos Clínicos, v. 2, n. 1, p. 59-71, 2009.

LIU, Y.; GUL, H.; ZHANG, J.; RAZA, J.; USMAN, M. Abusive Supervision and Suicidal Ideation: The Potential Role of Meaning in Life. Deviant Behavior, p. $1-12,2020$.

LOCKE, E. A.; SCHIPPERS, M. C. Improving lives: personal goal setting boosts student performance and happiness. Acad. Manag. Proc. 2018. 


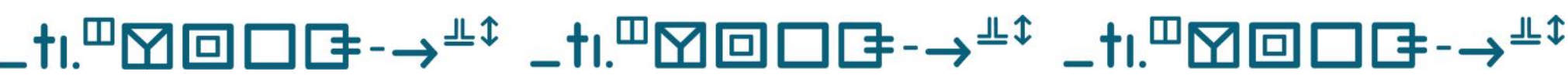

Bullying, apoio social e senso de vida: relato de discentes surdas

Valéria Maria Azevedo Guimarães • Karine David Andrade Santos et al.

LOPES, M. A. C.; LEITE, L. P. Concepções de surdez: a visão do surdo que se comunica em língua de sinais. Rev. Bras. Ed. Esp., v. 17, n.2, p. 305-320, 2011. MALTA, D. C.; MELLO, F. C. M.; PRADO, R. R.; SÁ, A. C. M. G. N.; MARINHO, F.; PINTO, I. V.; SILVA, M. M. A.; SILVA, M. A. I. Prevalência de bullying e fatores associados em escolares brasileiros. Ciênc. Saúde coletiva, v. 24, n. 4, 2019. MARCOLINO, E. C.; CAVALCANTE, A. L.; PADILHA, W. W. N.; MIRANDA, F. A. N.; CLEMENTINO; F. S. Bullying: prevalência e fatores associados à vitimização e à agressão no cotidiano escolar. Texto Contexto Enferm, v. 27, n. 1, p. 1-10, 2018.

MATIAS, R. C.; MARTINELLI, S. C. Um estudo correlacional entre apoio social e autoconceito de estudantes universitários. Avaliação (Campinas), v. 22, n. 1, p. 15-33, 2017.

MARTINS, S. E. S. O.; NAPOLITANO, C. J. Inclusão, acessibilidade e resistência: direitos de estudantes surdos à educação superior. Educar em Revista, v. 33, n. especial 3, p.107-126, 2017.

MELLO, F. C. M.; MALTA, D. C.; SANTOS, M. G.; SILVA, M. M. A.; SILVA, M. A. I. Evolução do relato de sofrer bullying entre escolares brasileiros: Pesquisa Nacional de Saúde Escolar - 2009 a 2015. Rev Bras Epidemiol, v. 21, p. 1-14, 2018.

MESQUITA, L. S. Políticas Públicas de Inclusão: o acesso da pessoa surda ao ensino superior. Educação \& Realidade, v. 43, n. 1, p. 255-273, 2018.

MILLEN, K.; DORN, B.; LUCKNER, J. L. Friendships and Self-Determination Among Students Who Are Deaf or Hard of Hearing. American Annals of the Deaf, v. 163, n. 5, p. 576-595, 2019.

MOELLER, M. P. Early intervention and language development in children who are deaf and hard of hearing. Pediatrics, v. 106, n. 3, p. 1-9, 2000. NEGRELLI, M. E. D.; MARCON, S. S. Família e criança surda. Ciência, Cuidado e Saúde, v. 5, n. 1, p. 98-107, 2006. 


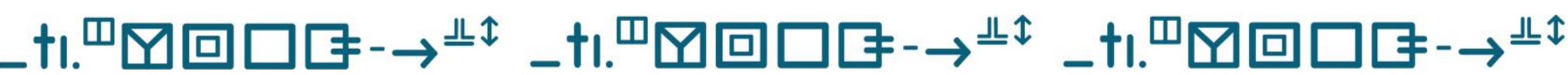

Bullying, apoio social e senso de vida: relato de discentes surdas

Valéria Maria Azevedo Guimarães • Karine David Andrade Santos et al.

NORWICH, B.; KELLY, N. Pupils' views on inclusion: Moderate learning difficulties and bullying in mainstream and special schools. British Educational Research Journal, v. 30, n. 1, p. 43-65, 2004.

NUNES, S. S.; SAIA, A. L.; SILVA, L. J.; MIMESSI, S. D. Surdez e educação: escolas inclusivas e/ou bilíngues? Revista Quadrimestral da Associação Brasileira de Psicologia Escolar e Educacional, v. 19, n. 3, p. 537-545, 2015.

ORGANIZAÇÃO DAS NAÇÕES UNIDAS PARA A EDUCAÇÃO, A CIÊNCIA E A CULTURA (UNESCO). Violência escolar e bullying: relatório sobre a situação mundial. Brasília, 54 p., 2019.

OLIVEIRA, K. G.; AQUINO, T. A. A. Logoterapia e cultura surda: Adaptação e validação do quationário sentido de vida para o contexto de pessoas surdas. Revista da Associação Brasileira de Logoterapia e Análise Existencial Logos \& existência, v. 3, n. 2, p. 238-254, 2014.

OLIVEIRA, W. A.; SILVA, M. A. I.; MELLO, F. C. M.; PORTO, D, L.; YOSHINAGA, A. C. M.; MALTA, D. C. Causas do Bullying: resultados da Pesquisa Nacional de saúde do escolar. Rev. Latino-Am. Enfermagem, p. 1-8, 2015.

PATIAS, N. D.; SIQUEIRA, A. C.; DIAS, A. C. G. Bater não educa ninguém! práticas educativas parentais coercitivas e suas repercussões no contexto escolar. Educ. Pesqui., v. 38, n. 04, p. 981-996, 2012.

PEREIRA, I. S. A Ética do Sentido de Vida: Fundamentos da Logoterapia. Aparecida, SP: Ideias e Letras, 2013.

PINQUART, M.; PFEIFFER, J. P. Bullying in students with and without hearing loss. Deafness \& Education International, 17, 101-110, 2015.

QUADROS, R. M.; KARNOPP, L. B. Língua de sinais brasileira: estudos linguísticos. Porto Alegre: Artmed, 2004.

QUADROS, R. M.; STUMPF, M. R. O primeiro curso de graduação em Letras Língua Brasileira de Sinais: educação à distância. ETD - Educação Temática Digital, v. 10, n. 2, p. 169-185, 2009. 


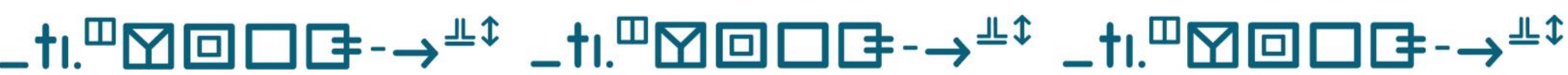

Bullying, apoio social e senso de vida: relato de discentes surdas

Valéria Maria Azevedo Guimarães • Karine David Andrade Santos et al.

RAMOS, D. A. S. M. Burnout, percepção de suporte social e autoeficácia em estudantes de ensino superior. Dissertação (Mestrado em Psicologia Aplicada) - Instituto de Psicologia da Universidade Federal de Uberlândia, 2015.

REINERT, M. Alceste, une méthodologie d'analyse des données textuelles et une application. AURÉLIA de $G$. de N. Bulletin de méthodologie sociologique, (28) 24-54, 1990.

RYAN, R. M. The peer group as a context for the development of young adolescent motivation and achievement. Child Development, v. 72, n. 4, p. 1135-1150, 2001.

SAMPAIO, J. M. C.; SANTOS, G. V.; OLIVEIRA, W. A.; SILVA, J. L.; MEDEIROS, M.; SILVA, M. A. J. Prevalência de bullying e emoções de estudantes envolvidos. Texto contexto enfermagem, v. 24, n. 2, p. 344-52, 2015.

SAMSSUDIN, S.; BARROS, A. B. Relação entre as crenças de auto-eficácia e o apoio social na transição para o trabalho em estudantes finalistas do ensino superior. Revista Psicologia, v. 25, n. 1, p. 159-171, 2011.

SANTANA, A. P. A inclusão do surdo no ensino superior no Brasil. Nasen. Journal of Research in Special Educational Needs, v. 16, n. 1, p. 85-88, 2016.

SANTOS, A. S.; OLIVEIRA, C. T.; DIAS, A. C. G. Características das relações dos universitários e seus pares: implicações na adaptação acadêmica. Revista Psicologia: Teoria e Prática, v. 17, n. 1, p. 150-163, 2015.

SARTORI, I. J. F. Políticas Públicas, Inclusão e Educação Bilíngue de surdos em Campinas (SP): 2007 - 2017. Dissertação (Mestrado em Educação) Programa de Pós-Graduação, Pontifícia Universidade Católica de Campinas. PUC Campinas, 2019. 


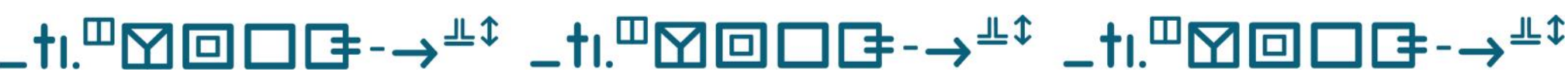

Bullying, apoio social e senso de vida: relato de discentes surdas

Valéria Maria Azevedo Guimarães • Karine David Andrade Santos et al.

SCHIPPERS, M. C.; MORISANO, D.; LOCKE, E. A.; SCHEEPERS, A. W.; LATHAM, G.; DE JONG, E. M. Conscious goal reflection boosts academic performance regardless of goal domain. Contemp. Educ. Psychol, 2019.

SCHIPPERS, M. C.; ZIEGLER, N. Life crafting as a way to find purpose and meaning in life. Frontiers in Psychology, 2019.

SHERIDAN, M. Inner lives of Deaf children: Interviews and analysis. Washington, DC: Gallaudet University Press, 2001.

SILVA, L. S.; BASTOS, T. Pais ouvintes e filhos surdos: impasses na comunicação. Entrelaçando - Revista Eletrônica de Cultura e Educação. Caderno temática: educação especial e inclusão, n, 8, p. 25-34, 2013.

SILVEIRA, D. R.; GRANDIM, F. J. Contribuições de Viktor FrankI ao movimento da saúde coletiva. Revista da abordagem gestáltica - Phenomenological Studies, v. 21, n. 2, p. 153-161, 2015.

SILVA, D.; TAVARES, E.; SILVA, E.; DUARTE, J.; CABRAL, L.; MARTINS, C. Vítimas e agressores - Manifestações de bullying em alunos do $6^{\circ}$ ao $9^{\circ}$ ano de escolaridade. Revista Portuguesa de Enfermagem de Saúde Mental, n. 5, p. 57-62, 2017.

SKLIAR, C. Um olhar sobre o nosso olhar acerca da surdez e das diferenças. In: A surdez: um olhar sobre as diferenças. 8. ed. Porto Alegre: Mediação, p. 5-32, 2016.

SMITH, K. L.; RUSH, L. L. Counseling students who are Deaf. In: LIPPINCOTT, J. A.; LIPPINCOTT, R. B. Special populations in college counseling: $A$ handbook for mental health professionals, Alexandria, VA: American Counseling Association, p. 231-245, 2007.

SOUZA, J. M. Bullying: uma das faces do preconceito homofóbico entre jovens no contexto escolar. Dissertação (Mestrado em Psicologia Social) Centro de Ciências de Educação e Ciências Humanas, Universidade Federal de Sergipe, São Cristóvão, 2013. 


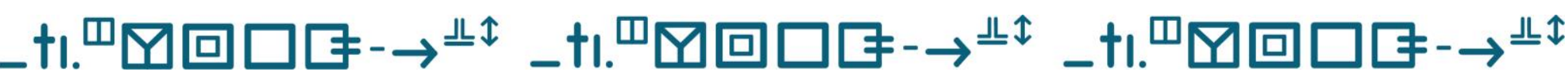

Bullying, apoio social e senso de vida: relato de discentes surdas

Valéria Maria Azevedo Guimarães • Karine David Andrade Santos et al.

SOUZA, L. C. Quando o bullying na escola afeta a vida adulta. Rev. Psicopedagogia, v. 36, n. 110, p. 153-62, 2019.

STELLING, E. P.; STELLING, L. F. P.; TORRES, E. M. S.; CASTRO, H. C. Pais ouvintes e filho surdo: dificuldades de comunicação e necessidade de orientação familiar. Espaço, n.42, 2014.

WAGNER, A.; FALCKE, D.; SILVEIRA, L. M. B. de O.; MOSMANN, C. P. A comunicação em famílias com filhos adolescentes. Psicologia em Estudo, 7(1), 75-80, 2002.

WARD, S. J.; KING, L. A. Work and the good life: How work contributes to meaning in life. Research in Organizational Behavior, v. 37, p. 59-82, 2017. WEINER, M. T., DAY, S. J.; GALVAN, D. Deaf and hard of hearing students' perspectives on bullying and school climate. American annals of the deaf, 158(3), 334-343, 2013.

WHITNEY, I.; SMITH, P. K.; THOMPSON, D. Bullying and children with special educational needs. In: Smith, P. K.; Sharp, S. School bullying: Insights and perspectives New York: Routledge, p. 213-240, 1994.

WHYTE, A. K.; GUIFFRIDA, D. A. Counseling Deaf College Students: The Case of Shea. Journal of College Counseling, v. 11, p. 184-192, 2008.

XAUSA, L. A. M. A Psicologia do Sentido de Vida. Vide Editorial, 2013.

ZAMPAR, J. A. S. Integração na universidade na percepção de estudantes com deficiência. Dissertação (Mestrado em educação) - Programa de PósGraduação em Educação Especial do Centro de Educação e Ciências Humanas, Universidade Federal de São Carlos. São Carlos, SP, 2015.

ZHANG, H.; CHEN, K.; CHEN, C.; SCHLEGEL, R. Personal aspirations, personenvironment fit, meaning in work, and meaning in life: A moderated mediation model. Journal of Happiness Studies, v. 20, n. 5, p. 1481-1497, 2019. 


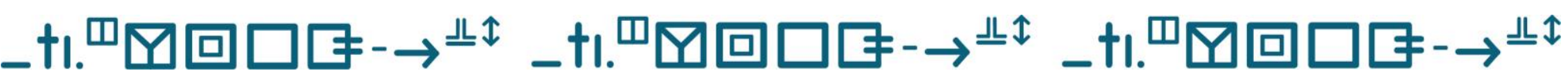

Bullying, apoio social e senso de vida: relato de discentes surdas

Valéria Maria Azevedo Guimarães • Karine David Andrade Santos et al.

\section{Publisher}

UNIVERSIDADE FEDERAL DE GOIÁS. CURSOS DE LETRAS: LIBRAS E DE LETRAS: TRADUÇÃO E INTERPRETAÇÃO EM LIBRAS/PORTUGUÊS DA FACUldade DE LETRAS/UFG. PuBlicaÇÃO NO PORTAL DE PERIÓdicos UFG. AS IDEIAS EXPRESSADAS NESTE ARTIGO SÃO DE RESPONSABILIDADE DE SEUS AUTORES, NÃO REPRESENTANDO, NECESSARIAMENTE, A OPINIÃO DOS EDITORES OU DA UNIVERSIDADE. 